\title{
Angiosarcoma: una rara lesión hepática
}

\section{Angiosarcoma: A Rare Hepatic Lesion}

\author{
Daniel Noreña-Rengifo ${ }^{10}$ Camilo Grondona-Ramírez ${ }^{1}$ Astrid Arroyave-Toro ${ }^{1,2}$
}

1 Universidad de Antioquia, Medellín, Antioquia, Colombia
2 Servicios de Salud San Vicente Fundación, Antioquia, Colombia

Rev Argent Radiol 2021;85:106-108.

Estimados editores,

El angiosarcoma hepático (AH), también llamado sarcoma de Kupffer, es una neoplasia maligna poco frecuente que corresponde a menos del $2 \%$ de los cánceres hepáticos, con predominio tres veces mayor en los hombres. Sin embargo, corresponde al tumor mesenquimatoso maligno más común del hígado. ${ }^{1-3}$

Se origina en la anastomosis de las células endoteliales con los canales vasculares y, aunque se han descrito algunos factores ambientales relacionados, no siempre es posible identificar un factor desencadenante. Su diagnóstico requiere de estudios imagenológicos, debido a que la clínica y las pruebas de laboratorio no presentan una característica representativa de la enfermedad.,3-6 El objetivo de este artículo es describir los hallazgos imagenológicos del $\mathrm{AH}$ y su correlación anatomopatológica.

Comunicamos el caso de un paciente masculino de 77 años, con antecedente de hipertensión arterial, diabetes mellitus tipo 2, tabaquismo activo y alcoholismo crónico, quien consultó por cuadro clínico de un mes de evolución de ictericia asociada a dolor abdominal difuso y pérdida de peso. Además, en los últimos días con coluria, melenas y hematemesis. En los exámenes paraclínicos se identificó anemia microcítica y trombocitopenia. Inicialmente, le realizaron una ecografía, que demostró múltiples lesiones hepáticas sugestivas de metástasis, por lo cual fue trasladado a nuestra institución, donde se realizaron endoscopia digestiva superior sin hallazgos patológicos y tomografía compuratizada (TC) de abdomen que demostró múltiples lesiones hipervasculares hepáticas (-Fig. 1). Teniendo en cuenta la clínica y los hallazgos hepáticos, se
Address for correspondence Brian Daniel Noreña Rengifo, Calle 70 \# 52-21, Medellín, Colombia (e-mail: brian.norena@udea.edu.co). realizó una biopsia percutánea, cuya patología diagnosticó AH (-Figs. 2 y 3 ).

El AH es considerado un tumor raro que está constituido por tejido endotelial y fibroblástico que prolifera y rodea los vasos. La mayoría de los casos ocurren entre la sexta y séptima década de la vida, aunque el rango de edades va desde la segunda a la octava década de la vida., 2,4,5,7

Los factores de riesgo asociados incluyen la exposición a cloruro de vinilo, Thorotrast ${ }^{\circledR}$ (dióxido de torio), estilbestrol y arsénico, el uso de anticonceptivos orales, consumo de esteroides anabólicos androgénicos y la radiación ionizante. También se ha asociado con el síndrome de Von Recklinghausen y la hemocromatosis, pero en la mayoría de los casos no se identifica un factor de riesgo claro. ${ }^{1-5,8,9}$

Las manifestaciones clínicas de la enfermedad son inespecíficas e incluyen: fatiga, malestar general, masa abdominal palpable, hepatomegalia, ictericia y dolor abdominal. En los exámenes de laboratorio se puede identificar alteración en las pruebas hepáticas, anemia y trombocitopenia. $^{1}$ En algunos casos se ha descrito un síndrome anémico ferropénico, anemia hemolítica microangiopática y trombocitopenia leve, similar a la encontrada en nuestro paciente. Debido al riesgo de hemorragia, aunque el diagnóstico definitivo es anatomopatológico, no se suele realizar dicha comprobación y el diagnóstico se determina con estudios imagenológicos. La ruptura espontánea del tumor con hemorragia intra-abdominal masiva se ha observado frecuentemente. ${ }^{1,4,5,9}$

Teniendo en cuenta que las imágenes suelen ser la primera aproximación diagnóstica, es importante reconocer sus principales características, pero debido a que received

April 28, 2020

accepted

October 29, 2020

published online

May 14, 2021
DOI https://doi.org/

10.1055/s-0041-1724051. ISSN 1852-9992.

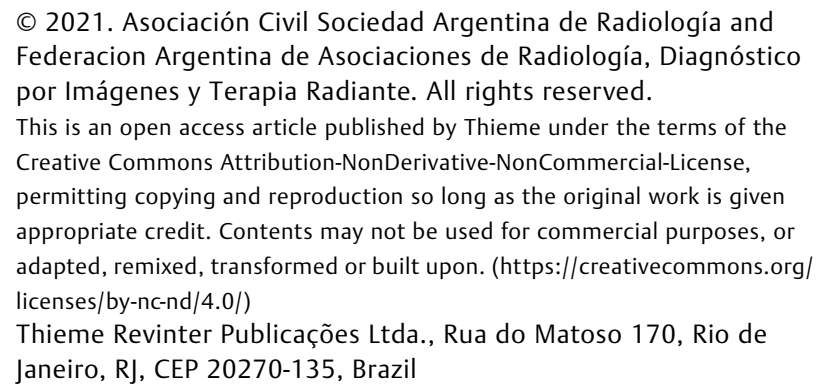

(C) 2021. Asociación Civil Sociedad Argentina de Radiología and Federacion Argentina de Asociaciones de Radiología, Diagnóstico por Imágenes y Terapia Radiante. All rights reserved.

This is an open access article published by Thieme under the terms of the Creative Commons Attribution-NonDerivative-NonCommercial-License, permitting copying and reproduction so long as the original work is given appropriate credit. Contents may not be used for commercial purposes, or adapted, remixed, transformed or built upon. (https://creativecommons.org/ licenses/by-nc-nd/4.0/)

Thieme Revinter Publicações Ltda., Rua do Matoso 170, Rio de Janeiro, RJ, CEP 20270-135, Brazil 

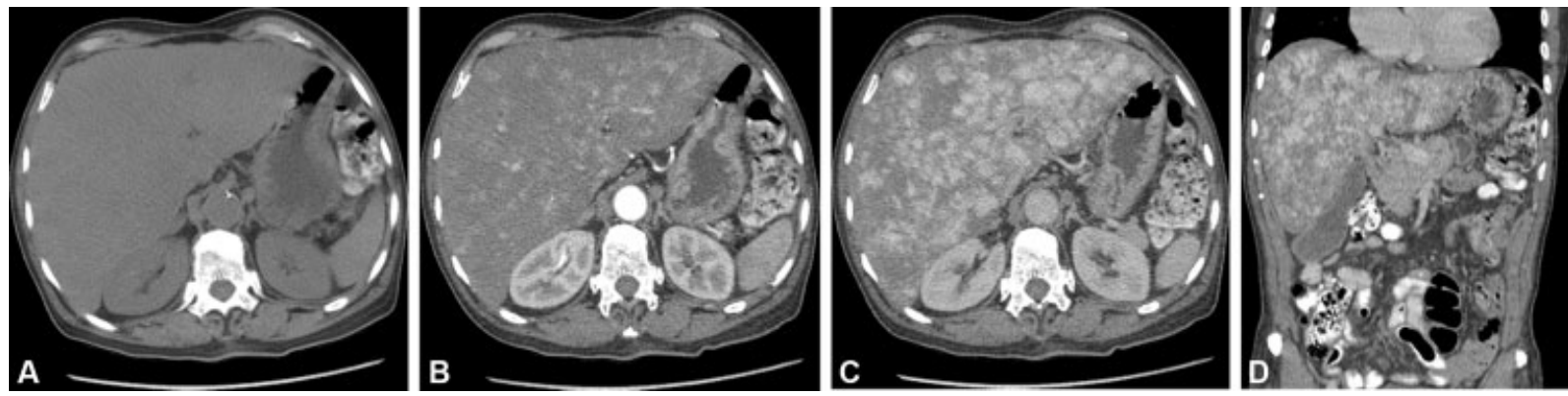

Fig. 1 TC de abdomen simple y contrastada. Hígado sin cambios por hepatopatía crónica, con lesiones hipervasculares difusas con realce heterogéneo y progresivo, que comprometen la totalidad del hígado. Su comportamiento se evidencia en las fases dinámicas, viendo como en la fase simple no es posible definir ninguna lesión (A), pero en la fase arterial (B) y portal (C, D) demuestran un patrón concordante con angiosarcoma hepático difuso.

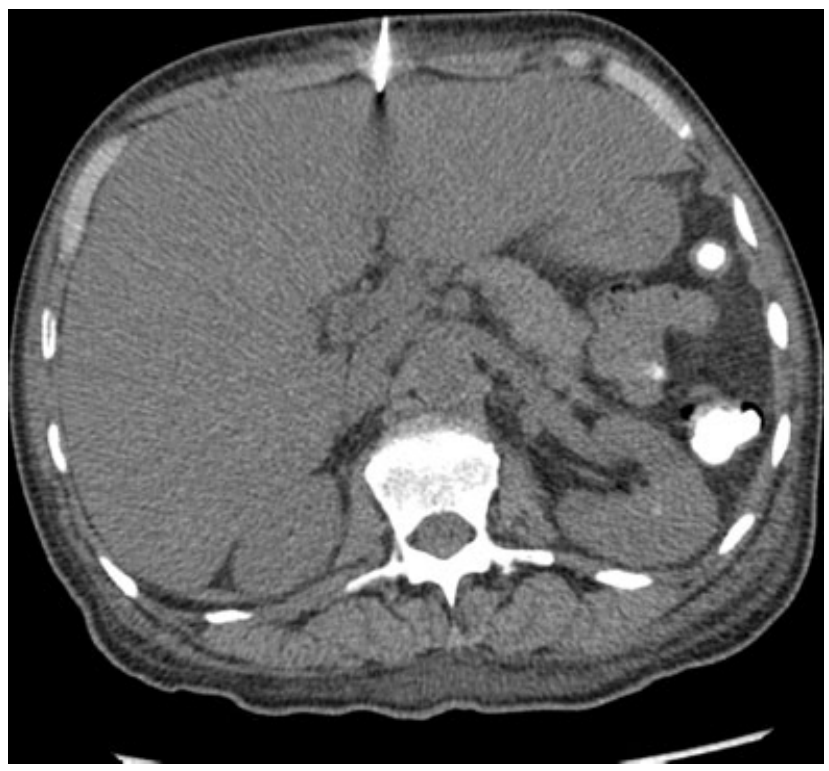

Fig. 2 Guía tomográfica para realización de biopsia percutánea en el lóbulo izquierdo.

es un tumor poco frecuente, las descripciones se basan en pocos reportes de casos. $^{8}$ Se han descrito tres principales patrones de presentación del tumor, tanto en TC como en resonancia magnética $(\mathrm{RM})$, como nódulos múltiples, masa dominante o lesión infiltrante difusa, esa última forma es la menos frecuente..$^{2-6,8} \mathrm{El}$ realce suele ser heterogéneo y progresivo, que se ha descrito clásicamente como anular en la fase arterial y con relleno centrípeto en la fase portal y de equilibrio, pero también puede ser centrífugo o con llenado inmediato. ${ }^{9}$ En los estudios sin medio de contraste se pueden identificar áreas de hemorragia. ${ }^{2-6,8}$

La RM permite caracterizar mejor los componentes internos de la lesión, encontrando típicamente una lesión heterogénea, con intensidad de señal variable tanto en las secuencias potenciadas en $\mathrm{T} 1$ como en $\mathrm{T} 2$, que se correlacionan con el componente hemorrágico. ${ }^{3}$ Los hallazgos en la ecografía son inespecíficos, por lo cual siempre es necesaria la caracterización mediante TC o RM. ${ }^{3}$

La tomografía con emisión de positrones (PET-TC) ha sido usada para determinar la presencia de metástasis, pero no tiene tanto valor en el diagnóstico como tal del $\mathrm{AH}^{5}{ }^{5}$

Dentro de los diagnósticos diferenciales se deben considerar los hemangiomas, los hemangioendoteliomas epitelioides, las metástasis hipervasculares y los hepatocarcinomas atípicos. ${ }^{5,6,10}$ El principal de ellos es el hemangioma y especialmente el hemangioma cavernoso, con el cual el diagnóstico diferencial se hace porque la atenuación de la lesión suele ser similar a la de los grandes vasos (aorta) en todas las fases, de manera más organizada y con contornos mejor definidos que el AH. ${ }^{5,6}$

Dada su agresividad y altas tasas de metástasis tempranas, se relaciona con un pobre pronóstico, que puede variar entre 6 meses y 2 años aproximadamente. La mayoría de los pacientes mueren un año después del diagnóstico, usualmente por un diagnóstico tardío. ${ }^{2,4,5,7}$ Al momento del diagnóstico, la mayoría de los pacientes presentan metástasis, usualmente pulmonares, esplénicas u óseas, lo cual empeora su pronóstico. ${ }^{3,7}$ El tratamiento depende del estadio, pero se considera que no es curativo, sino con
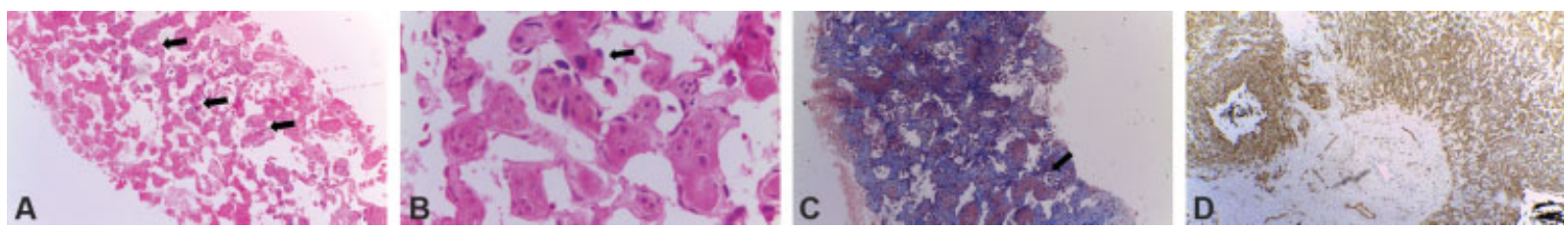

Fig. 3 Patología con coloraciones con hematoxilina y eosina (H\&E) e inmunohistoquímica CD34. En la imagen con H\&E a 4x se identifica una neoplasia vascular maligna que altera la arquitectura normal del hígado, con espacios vasculares revestidos por células endoteliales neoplásicas (flechas en A). En la misma coloración con H\&E con 40x se identifican espacios vasculares malformados y revestidos por células endoteliales atípicas (flecha en B). La coloración de histoquímica con tricrómico (C) y la inmunohistoquímica para CD34 (D) destacan los espacios vasculares que constituyen la neoplasia (flecha en C). 
medidas paliativas. La cirugía es el tratamiento de elección cuando la lesión es resecable y, aunque puede mejorar la supervivencia y acompañarse de quimioterapia, la recurrencia es frecuente. ${ }^{1,4,5}$

\section{Responsabilidades éticas}

Protección de personas y animales: Los autores declaran que para esta investigación no se han realizado experimentos en seres humanos ni en animales.

Confidencialidad de los datos: Los autores declaran que en este artículo no aparecen datos de pacientes.

Derecho a la privacidad y consentimiento informado: Los autores declaran que en este artículo no aparecen datos de pacientes y se cuenta con su consentimiento informado.

Conflictos de intereses

Los autores declaran no tener conflictos de intereses.

\section{Referencias}

1 Kim HR, Rha SY, Cheon SH, Roh JK, Park YN, Yoo NC. Clinical features and treatment outcomes of advanced stage primary hepatic angiosarcoma. Ann Oncol. 2009;20(04):780-787

2 Kim B, Byun JH, Lee JH, Park BJ, Kwon HJ, Lee JH, et al. Imaging findings of primary hepatic angiosarcoma on gadoxetate disodium-enhanced liver MRI: comparison with hepatic haemangiomas of similar size. Clin Radiol. 2018;73(03): 244-253

3 Koyama T, Fletcher JG, Johnson CD, Kuo MS, Notohara K, Burgart LJ. Primary hepatic angiosarcoma: findings at CT and MR imaging. Radiology. 2002;222(03):667-673

4 Egea Valenzuela J, López Poveda MJ, Pérez Fuenzalida FJ, Garre Sánchez C, Martínez Barba E, Carballo Álvarez F. Angiosarcoma hepático. Presentación de dos casos. Rev Esp Enferm Dig. 2009; 101(06):434-437

5 Thapar S, Rastogi A, Ahuja A, Sarin S. Angiosarcoma of the liver: imaging of a rare salient entity. J Radiol Case Rep. 2014;8(08):24-32

6 Peterson MS, Baron RL, Rankin SC. Hepatic angiosarcoma: findings on multiphasic contrast-enhanced helical CT do not mimic hepatic hemangioma. AJR Am J Roentgenol. 2000;175(01):165-170

7 Chien C, Hwang C, Yeh C, Chen H, Wu J, Chan S, et al. Liver angiosarcoma, a rare liver malignancy, presented with intrabdominal bleeding due to rupture-a case report. World J Surg Oncol. 2012;10:23

8 Arteche E, Ostiz S, de Miguel C. Angiosarcoma hepático difuso secundario al depósito de thorotrast. Radiologia (Madr). 2007;49 (01):57

9 Pickhardt PJ, Kitchin D, Lubner MG, Ganeshan DM, Bhalla S, Covey AM. Primary hepatic angiosarcoma: multi-institutional comprehensive cancer centre review of multiphasic CT and MR imaging in 35 patients. Eur Radiol. 2015;25(02):315-322

10 Shiba H, Sakamoto T, Iida T, Furukawa K, Haruki K, Yanaga K. Hepatic angiosarcoma mimicking hepatic epithelioid hemangioendothelioma: report of a case. Int Cancer Conf J. 2016;6(01):29-34 